\title{
Standing and travelling waves in the shallow-water circular hydraulic jump
}

\author{
Arnab K. Ray* \\ Inter-University Centre for Astronomy and Astrophysics \\ Post Bag 4, Ganeshkhind, Pune University Campus \\ Pune 411007, Indid
}

Jayanta K. Bhattacharje田

Department of Theoretical Physics

Indian Association for the Cultivation of Science

Jadavpur, Kolkata 700032, India

(Dated: November 13, 2018)

\begin{abstract}
A wave equation for a time-dependent perturbation about the steady shallow-water solution emulates the metric an acoustic white hole, even upon the incorporation of nonlinearity in the lowest order. A standing wave in the sub-critical region of the flow is stabilised by viscosity, and the resulting time scale for the amplitude decay helps in providing a scaling argument for the formation of the hydraulic jump. A standing wave in the super-critical region, on the other hand, displays an unstable character, which, although somewhat mitigated by viscosity, needs nonlinear effects to be saturated. A travelling wave moving upstream from the sub-critical region, destabilises the flow in the vicinity of the jump, for which experimental support has been given.
\end{abstract}

PACS numbers: 47.35.Bb, 47.15.Cb, 47.32.Ff

Keywords: Gravity waves, Laminar boundary layers, Separated flows

\section{INTRODUCTION}

The hydraulic jump is a subject of textbooks in fluid dynamics [1, 2, 3, 4, 5]. With a jet of water impinging vertically on a flat surface, it can be seen that from the point of impingement, the water spreads out radially in a thin film, and beyond a certain distance, the height of the fluid layer abruptly increases, exhibiting the circular hydraulic jump [6, 7, 8]. This is a common occurrence in a kitchen sink, and somewhat differently this phenomenon is also exhibited as a tidal bore moving upstream in a river [4], which makes the hydraulic jump an engineering problem of considerable practical interest as well.

Viscosity of the flowing liquid is the key factor that makes the hydraulic jump possible [9, 10, 11], and in consequence of this, a scaling relation for the hydraulic jump radius has been derived by Bohr et al. [9], with strong experimental support coming subsequently in favour of this scaling relation through a work by Hansen et al. [12]. Some later works have also shown that surface tension has an involvement with the jump in shallow-layer flows [13, 14, 15]. While dwelling on these matters, it would be very pertinent to mention that in a recently published work, Rolley et al. [15] have brought forth an interesting experimental study in which the hydraulic jump was seen to form in the flow of liquid helium, below its superfluid transition temperature. The radius of the jump and the depth profile of the flow were also not altered appreciably at the superfluid transition, as though the fluid was still normal. This feature is probably due to the fact that the flow velocity in this situation is supercritical with respect to the critical velocity for superfluidity.

While it is usual to address all jump-related issues in the stationary regime, in a departure from this practice, explicit timedependence in the shallow-water approach to the circular hydraulic jump has been considered in this paper. A time-dependent perturbation has been imposed on the steady volumetric flow rate. As an immediate and interesting consequence of this line of attack, a linearised equation of motion for the perturbation has been found to have a mathematical form that is very much like the metric of an acoustic white hole. This compatibility makes it possible to match the critical properties of the flow with the horizon condition of the acoustic white hole, with important consequences leading to the formation of the jump itself.

As regards the stability of stationary solutions, it has been shown that when the perturbation is treated as a standing wave, bounded at two separate spatial points in the sub-critical flow region, viscosity will cause a damping of the amplitude of the perturbation. A resultant time scale for viscous damping of the perturbation has been argued to be the same time scale on which the flow is dissipatively slowed down by viscosity, and using this time scale, along with the analogy of a white hole, it becomes possible to establish a physical explanation for the standard scaling relation of the hydraulic jump radius [9]. A study to this effect has been reported before [16] for the one-dimensional channel flow.

\footnotetext{
*Electronic address: akr@iucaa.ernet.in

${ }^{\dagger}$ Also at: Harish-Chandra Research Institute, Chhatnag Road, Jhunsi, Allahabad 211019, India

${ }^{\ddagger}$ Electronic address: tpjkb@mahendra.iacs.res.in
} 
In the super-critical region, however, the standing wave does not exhibit similarly stable behaviour. Viscosity goes some way in opposing the growth of the perturbation in this region, but this divergent aspect of the perturbation has to be saturated ultimately by the conspicuously nonlinear effects in the super-critical region of the flow. This feature is in keeping with what has been seen from earlier experiments [17]. A very intriguing feature that has emerged on including nonlinearity is that there is no alteration of the conditions under which it is possible to construct an acoustic metric for the flow.

As opposed to standing waves, treating the perturbation as a travelling wave leads to a different conclusion about the stability of the flow. In this case it can be seen that the outgoing mode of the travelling wave has a growing amplitude in the super-critical region of the flow, but it ultimately decays out in the sub-critical flow region. On the other hand, the incoming mode causes great disturbance in the flow as it propagates upstream in the sub-critical region, especially very close to the jump. Experimental evidence has been adduced for the latter kind of behaviour [18].

\section{A WAVE EQUATION AND THE METRIC OF AN ACOUSTIC WHITE HOLE}

The flow is described by the Navier-Stokes equation and the equation of continuity, with both of them modified for a shallow radial flow confined to a plane [9]. The steady flow variables would be the velocity of the flow, $v(r)$, and the local height of the incompressible fluid, $h(r)$, with the former having been obtained in the shallow-water theory by vertically averaging the radial component of the velocity, $u(r, z)$, over the height, $h(r)$. The boundary conditions for the averaging are $u(r)=0$ at $z=0$, and $\partial u / \partial z=0$ at $z=h(r)$, under the assumption that while the vertical velocity is much small compared with the radial velocity, the vertical variation of the velocity (through the shallow layer of water) is much greater than its radial variation [9].

The dynamic generalisation of both $v$ and $h$, and their corresponding dynamic equations may be written as

$$
\frac{\partial h}{\partial t}+\frac{1}{r} \frac{\partial}{\partial r}(r v h)=0
$$

and

$$
\frac{\partial v}{\partial t}+v \frac{\partial v}{\partial r}+g \frac{\partial h}{\partial r}=-\nu \frac{v}{h^{2}}
$$

respectively, with the usual viscous term in the Navier-Stokes equation having been suitably approximated in Eq. (2), following the arguments offered by Bohr et al. [9]. The steady solutions of Eqs. (1) and (2) above lead to the phenomenon of the hydraulic jump.

To carry out a linearised perturbative analysis, it will be convenient to work with a new variable that is defined as $f=r v h$. The wisdom behind this choice may be discerned from the structure of Eq. (11), the steady solution of which can be integrated to give $r v h=Q / 2 \pi$, with the integration constant $Q$ being the steady volumetric flow rate. Therefore, the new variable $f$ can be physically identified with the time-dependent volumetric flow rate, and its steady solution will be a constant.

Solutions of the form $v(r, t)=v_{0}(r)+v^{\prime}(r, t)$ and $h(r, t)=h_{0}(r)+h^{\prime}(r, t)$ are to be used, in which the subscript 0 indicates steady solutions, while the primed quantities are time-dependent perturbations about those steady solutions. Linearising in these fluctuating quantities gives the fluctuation of $f$ about its constant steady value, $f_{0}=r v_{0} h_{0}=Q / 2 \pi$, as

$$
f^{\prime}=r\left(v_{0} h^{\prime}+h_{0} v^{\prime}\right)
$$

In terms of $f^{\prime}$, it is possible to write from Eq. (1),

$$
\frac{\partial h^{\prime}}{\partial t}=-\frac{1}{r} \frac{\partial f^{\prime}}{\partial r}
$$

which, in conjunction with Eq. (3), gives,

$$
\frac{\partial v^{\prime}}{\partial t}=\frac{1}{r h_{0}}\left(\frac{\partial f^{\prime}}{\partial t}\right)+\frac{v_{0}}{r h_{0}}\left(\frac{\partial f^{\prime}}{\partial r}\right)
$$

A further partial differentiation of Eq. (5) with respect to $t$, followed by extracting the linearised terms in $v^{\prime}$ and $h^{\prime}$ from Eq. (2), and substitution of these perturbed quantities in terms of $f^{\prime}$, will deliver

$$
\frac{\partial}{\partial t}\left[v_{0}\left(\frac{\partial f^{\prime}}{\partial t}\right)\right]+\frac{\partial}{\partial t}\left[v_{0}^{2}\left(\frac{\partial f^{\prime}}{\partial r}\right)\right]+\frac{\partial}{\partial r}\left[v_{0}^{2}\left(\frac{\partial f^{\prime}}{\partial t}\right)\right]+\frac{\partial}{\partial r}\left[v_{0}\left(v_{0}^{2}-g h_{0}\right) \frac{\partial f^{\prime}}{\partial r}\right]=-\nu \frac{v_{0}}{h_{0}^{2}}\left(\frac{\partial f^{\prime}}{\partial t}+3 v_{0} \frac{\partial f^{\prime}}{\partial r}\right) .
$$

It should be instructive to examine Eq. (6) in its inviscid limit, i.e. when $\nu=0$. Some studies [19, 20, 21] have revealed a close analogy between the propagation of a wave in a moving fluid and of light in curved space-time. On this particular issue, 
Schützhold and Unruh have shown that gravity waves in a shallow layer of liquid are governed by the same wave equation as for a scalar field in curved space-time [19]. For an inviscid, incompressible and irrotational flow, the flow velocity has been defined to be the gradient of a scalar potential. Perturbing this potential about its background value, under the restricted condition of the background flow height being a constant, leads to an effective metric, in which the velocity of gravity waves replace the speed of sound in sonic analogues which closely reflect features seen in general relativistic studies [19, 20].

The present analysis is devoted to what is essentially a dissipative system (since it includes viscosity). However, in its inviscid limit, this system will deliver the same metric obtained by Schützhold and Unruh from their purely inviscid model. It must be stressed here that the choice of perturbing the constant background flow rate is paticularly expedient, since conservation of matter holds good even in a system that undergoes viscous dissipation. It is to be further noted that the background velocity and flow height in this treatment are in general stationary functions of space. Extracting the inviscid terms only from Eq. (6) by setting $\nu=0$, it should be easy to ultimately render these terms into a compact formulation that looks like [20]

$$
\partial_{\alpha}\left(f^{\alpha \beta} \partial_{\beta} f^{\prime}\right)=0
$$

in which the Greek indices run from 0 to 1 , with the identification that 0 stands for $t$ and 1 stands for $r$. On inspecting the terms in the left hand side of Eq. (6), the symmetric matrix,

$$
\mathrm{f}^{\alpha \beta}=v_{0}\left[\begin{array}{cc}
1 & v_{0} \\
v_{0} & v_{0}^{2}-g h_{0}
\end{array}\right]
$$

can be obtained. It is well known that in terms of the metric $\mathrm{g}_{\alpha \beta}$, the d'Alembertian for a scalar in curved space, is given by [20]

$$
\triangle \psi \equiv \frac{1}{\sqrt{-\mathrm{g}}} \partial_{\alpha}\left(\sqrt{-\mathrm{g}} \mathrm{g}^{\alpha \beta} \partial_{\beta} \psi\right)
$$

in which $\mathrm{g}^{\alpha \beta}$ is the inverse of the matrix implied by $\mathrm{g}_{\alpha \beta}$. Under the equivalence that $\mathrm{f}^{\alpha \beta}=\sqrt{-\mathrm{g}} \mathrm{g}^{\alpha \beta}$, and therefore, $\mathrm{g}=$ $\operatorname{det}\left(f^{\alpha \beta}\right)$, an effective metric can immediately be set down as

$$
\mathrm{g}_{\text {eff }}^{\alpha \beta}=\left[\begin{array}{cc}
1 & v_{0} \\
v_{0} & v_{0}^{2}-g h_{0}
\end{array}\right],
$$

which is entirely identical to the one derived by Schützhold and Unruh. By matrix inversion the inverse effective metric, $\mathrm{g}_{\alpha \beta}^{\text {eff }}$, is easily obtained from Eq. (10). For this system, in which the spatial dependence is only on the radial coordinate, the critical condition, $v_{0}^{2}=g h_{0}$, is also identified precisely as the horizon condition of either an acoustic black hole or an acoustic white hole, depending on the direction of the flow. The critical condition for a flow progressing from the super-critical region to the sub-critical region, is analogous to the horizon of an acoustic white hole.

In presenting the perturbative analysis in this paper, however, viscosity has been included in the governing equations, to finally arrive at Eq. (6). The presence of viscosity disrupts the precise symmetry of the inviscid conditions described by Eq. (7). This implies that the clear-cut horizon condition, obtained from the inviscid limit, will be affected. However, this effect, for small viscosity, as Schützhold and Unruh have argued, cannot be too drastic. They treated viscosity as a small adjunct effect on the inviscid flow, and concluded that the basic properties of gravity waves will not be affected overmuch. One way or the other, the most important feature to emerge from the analogy of a white hole horizon shall remain qualitatively unchanged, namely, that a disturbance propagating upstream from the sub-critical flow region (where $v_{0}^{2}<g h_{0}$ ) cannot penetrate through the horizon into the super-critical region of the flow (where $v_{0}^{2}>g h_{0}$ ). In keeping with this, Volovik has also pointed out that the jump condition can be closely related to the horizon of a white hole [21], a surface that nothing can penetrate. This property of the flow has a crucial bearing on the way the hydraulic jump is formed.

It has also been intriguing to note that the linearised perturbation dynamics in the shallow-water hydraulic jump is remarkably identical to the corresponding dynamics of astrophysical accretion on to an accretor (either a star or any compact object) [22, 23, 24, 25], with the only important point of difference being that whereas in the former case a disturbance in the fluid propagates as surface gravity waves, in the latter case a disturbance propagates as acoustic waves. In all other important qualitative respects, the similarities between what is an incompressible two-dimensional hydraulic jump outflow taking place on the laboratory scale, and what is a compressible spherically symmetric accretion inflow happening on the astrophysical scale, are very much unexpected. Apropos of this matter, however, Landau and Lifshitz have made a general but very significant observation that "there is a remarkable analogy between gas flow and the flow in a gravitational field of an incompressible fluid with a free surface, when the depth of the fluid is small..." [2].

\section{STANDING WAVES IN THE SUB-CRITICAL REGION AND SCALING ARGUMENTS}

The perturbation is treated as a standing wave, which is constrained to vanish at two separate boundaries. Between these two boundaries the flow has to be continuous. Since the jump is a discontinuity in the flow, the boundaries of the standing 
wave perturbation will have to be chosen on one side of the jump only, although Eq. (6) itself holds true over the entire flow space. Therefore, the stability analysis is confined to the sub-critical region of the flow only, where $v_{0}<c_{\mathrm{g}}$, with $c_{\mathrm{g}}$ being the steady value of the speed of surface gravity waves, expressible as $c_{\mathrm{g}}^{2}=g h_{0}$. As for the boundaries of the perturbation, one of them can be the outer boundary of the steady flow itself, where, by virtue of the boundary condition on the steady flow, the perturbation would naturally decay out. The inner boundary on the other hand, may be chosen to be infinitesimally close to the jump, which can be conceived of as a standing shock front. Going by the analogy of a white hole, this point will be seen as an impenetrable surface by any disturbance in the sub-critical region, and, therefore, the amplitude of the standing wave will decay in its neighbourhood. Between these two boundaries (which actually define the entire sub-critical flow region) the flow would have entirely lost its laminar character, and it would be most suited to deriving some physical insight about the behaviour of the perturbation and the influence of viscosity on it.

Upon using a solution of the form $f^{\prime}(r, t)=p_{\omega}(r) \exp (-\mathrm{i} \omega t)$, a quadratic equation in $\omega$ is obtained, and it is given by

$$
-\omega^{2} p_{\omega}-\mathrm{i} \omega\left[2 \frac{\mathrm{d}}{\mathrm{d} r}\left(v_{0} p_{\omega}\right)-\nu \frac{p_{\omega}}{h_{0}^{2}}\right]+\frac{1}{v_{0}} \frac{\mathrm{d}}{\mathrm{d} r}\left[v_{0}\left(v_{0}^{2}-c_{\mathrm{g}}^{2}\right) \frac{\mathrm{d} p_{\omega}}{\mathrm{d} r}\right]+3 \nu \frac{v_{0}}{h_{0}^{2}} \frac{\mathrm{d} p_{\omega}}{\mathrm{d} r}=0 .
$$

This expression is first multiplied by $v_{0} p$ and, between the two chosen boundaries, an integration by parts is carried out. Imposing the requirement that all integrated "surface" terms are to vanish at the two boundaries, will finally give

$$
\omega^{2} \int v_{0} p_{\omega}^{2} \mathrm{~d} r+\mathrm{i} \omega \nu \int \frac{v_{0} p_{\omega}^{2}}{h_{0}^{2}} \mathrm{~d} r+\int v_{0}\left(v_{0}^{2}-c_{\mathrm{g}}^{2}\right)\left(\frac{\mathrm{d} p_{\omega}}{\mathrm{d} r}\right)^{2} \mathrm{~d} r-3 \nu \int\left(\frac{v_{0}}{h_{0}}\right)^{2} p_{\omega} \frac{\mathrm{d} p_{\omega}}{\mathrm{d} r} \mathrm{~d} r=0
$$

A solution for $\omega$ can be found by solving the foregoing quadratic equation. Of immediate interest is the real part of the perturbation, going as

$$
\Re(-\mathrm{i} \omega)=-\frac{\nu}{2}\left[\int \frac{v_{0} p_{\omega}^{2}}{h_{0}^{2}} \mathrm{~d} r\right]\left[\int v_{0} p_{\omega}^{2} \mathrm{~d} r\right]^{-1} .
$$

In the sub-critical region the flow height, $h_{0}(r)$, is very weakly dependent on $r$, and so $\Re(-\mathrm{i} \omega) \simeq-\nu / 2 h_{0}^{2}$. Under inviscid conditions, the perturbation will have a constant amplitude in time, but viscosity, with all its dissipative implications, causes the perturbation to decay out. This decay of the amplitude of the perturbation will go as $\exp \left(-\nu t / 2 h_{0}^{2}\right)$, and it will also set up a time scale for viscosity to have its dissipative effect, which, to an order-of-magnitude, will be given by $t_{\mathrm{visc}} \sim h_{0}^{2} / \nu$.

The time scale on which viscous drag in the fluid will cause a dissipative slowing down of the flow, will also be given by $t_{\mathrm{visc}}$. The information of an advanced layer of fluid slowing down has to propagate upstream to preserve the smooth continuity of the fluid flow. However, this propagation can happen no faster than the speed of surface gravity waves, $c_{\mathrm{g}}$, and in the region where $v_{0}>c_{\mathrm{g}}$, no information, therefore, can propagate upstream [2, 11]. So a stream of fluid that has arrived later, moves on ahead, unhindered and uninformed, till its speed becomes comparable with the speed of the surface gravity waves, and only then does any information about a "barrier" ahead catches up with the fluid. At this stage one may define a dynamic time scale, $t_{\mathrm{dyn}} \sim r / v_{0}$, which is the time scale on which the bulk flow proceeds. Setting $t_{\mathrm{visc}} \simeq t_{\mathrm{dyn}}$ with the additional requirement that $v_{0} \simeq c_{\mathrm{g}}$, gives a condition for the "news" of the viscous slowing down finally catching up with the bulk flow itself. The continuity equation gives a further constraint that $r v_{0} h_{0}=Q / 2 \pi$, and on using this, a scaling behaviour for the hydraulic jump radius,

$$
r_{\mathrm{j}} \sim Q^{5 / 8} \nu^{-3 / 8} g^{-1 / 8}
$$

is obtained. This is exactly the scaling relation obtained by Bohr et al. [9], a result that was experimentally corroborated later by Hansen et al. [12].

The crucial factor that emerges from the above discussion is that for the formation of the hydraulic jump, the two time scales, $t_{\mathrm{visc}}$ and $t_{\mathrm{dyn}}$, would have to match each other closely, when the Froude number, $\mathcal{F}$, is close to unity. Under these conditions, a layer of fluid arriving late is confronted by a barrier formed by a layer of fluid moving ahead with an abrupt slowness. This slowly moving layer of fluid flowed past earlier in time, and at far radial distances it has been retarded considerably by viscous drag. In this situation there could not be an indefinite accumulation of the fluid, and since continuity of the fluid flow has to be preserved, the newly arrived fluid layer slides over the earlier viscosity dragged slowly moving layer of fluid, and what is seen is a sudden increase in the height of the fluid layer, a phenomenon that is known as a hydraulic jump. It is conceivable that this picture also explains the eddies which form immediately after the jump [12].

\section{STANDING WAVES IN THE SUPER-CRITICAL REGION AND THE ROLE OF NONLINEARITY}

So far the mathematical treatment was concerned primarily with developing and explaining a linearised perturbative effect on a steady background solution of the flow. However, what is actually being studied is a fluid dynamical system after all, and 
to derive a complete understanding of this flow system, at one stage or the other, it should be necessary to address the issue of nonlinearity. It has been established through some earlier experimental and theoretical works [17, 26] that the stability of the flow in the super-critical region comes under threat, and in this connection it is generally recognised that in one way or the other nonlinearity has an involvement.

In this context the question that has to be first posed is about the possibility of carrying out a self-consistent perturbative analysis that encompasses nonlinearity. Going back to the earlier prescription of $v(r, t)=v_{0}(r)+v^{\prime}(r, t)$ and $h(r, t)=$ $h_{0}(r)+h^{\prime}(r, t)$, but retaining the nonlinear term in the definition of $f^{\prime}$, it is easy to show that $f^{\prime}=r\left(v_{0} h^{\prime}+h_{0} v^{\prime}+v^{\prime} h^{\prime}\right)$, while

$$
\frac{\partial h^{\prime}}{\partial t}=-\frac{1}{r} \frac{\partial f^{\prime}}{\partial r}
$$

and

$$
\frac{\partial v^{\prime}}{\partial t}=\frac{1}{r h}\left(\frac{\partial f^{\prime}}{\partial t}\right)+\frac{v}{r h}\left(\frac{\partial f^{\prime}}{\partial r}\right)
$$

The two foregoing results are mathematically exact, and on comparing them with Eqs. (4) and (5), they are seen to be rather intriguing too. They show that regardless of the nature of the background flow - stationary or otherwise - the mathematical interdependence among $v^{\prime}, \rho^{\prime}$ and $f^{\prime}$ retain an invariant form. This leads, as it shall be presently demonstrated, to some interesting consequences so far as nonlinearity is concerned. Following the principle behind the derivation of Eq. (6), it can be shown that on retaining the lowest order of nonlinearity it is possible to arrive at a similar relation that is of the form

$$
\left(\mathcal{L}_{0}+\lambda \mathcal{L}_{1}\right) f^{\prime}=-\nu \frac{v_{0}}{h_{0}^{2}}\left\{\left(1-3 \lambda \frac{h^{\prime}}{h_{0}}\right) \frac{\partial f^{\prime}}{\partial t}+3 v_{0}\left[1+\lambda\left(\frac{v^{\prime}}{v_{0}}-3 \frac{h^{\prime}}{h_{0}}\right)\right] \frac{\partial f^{\prime}}{\partial r}\right\},
$$

in which $\mathcal{L}_{0}$ is the operator implied by Eqs. (7) and (8), and $\lambda$ is a "switch" parameter which can assume values of 0 and 1 only, depending, respectively, on whether one does not or does want to retain nonlinearity in the lowest order. The most interesting aspect of Eq. (17), however, is to be seen in $\mathcal{L}_{1}$, which is an operator that derives solely from accounting for lowest-order nonlinearity (i.e. with fluctuations upto the second order only). The structure of $\mathcal{L}_{1}$ is exactly similar to the form of $\mathcal{L}_{0}$, as Eq. (7) gives it, with the only difference being that the corresponding metric elements, referred to in this case as $\tilde{f}^{\alpha \beta}$, will involve time-dependent first-order fluctuations, unlike the case of the stationary background state, given by Eq. (8). Expressed fully these metric elements will read as

$$
\tilde{\mathbf{f}}^{\alpha \beta}=-v_{0}\left[\begin{array}{cc}
\tilde{h} & v_{0} \tilde{h}-v^{\prime} \\
v_{0} \tilde{h}-v^{\prime} & v_{0}^{2} \tilde{h}-2 v_{0} v^{\prime}
\end{array}\right]
$$

in which $\tilde{h}=h^{\prime} / h_{0}$, and the Greek indices, $\alpha$ and $\beta$, both run from 0 to 1 , as before. The fundamentally symmetric form of $\tilde{\mathrm{f}}^{\alpha \beta}$ can only mean that even with nonlinearity incorporated in the treatment, in the inviscid limit (i.e. for $\nu=0$ ), it should be very much possible to construct an analogue acoustic black hole metric (or white hole metric, depending on the direction of the flow), following the mathematical procedure adopted earlier in Section $\Pi$ Going by the form of Eqs. (15) and (16), it is also worth surmising at this point that this invariance will not be disturbed even when nonlinearity upto any higher order might be accounted for.

Another aspect of Eq. (17) is that apart from nonlinearity it also incorporates viscosity. The question is to what extent viscosity is important in determining the fate of a standing wave perturbation in the super-critical region. Going back to Eq. (12), but in this instance imposing the requirement that the standing wave will be bounded within the super-critical region only, close to the jump itself and at a point very close to the origin of the radial flow, it will be easy to see that for $v_{0}>c_{\mathrm{g}}$, there will be a growing mode for the perturbation. A condition for this instability can be set down as

$$
\int\left[v_{0}\left(v_{0}^{2}-c_{\mathrm{g}}^{2}\right)\left(\frac{\mathrm{d} p_{\omega}}{\mathrm{d} r}\right)^{2}-3 \nu\left(\frac{v_{0}}{h_{0}}\right)^{2} p_{\omega} \frac{\mathrm{d} p_{\omega}}{\mathrm{d} r}\right] \mathrm{d} r>0,
$$

whose form implies that if viscosity, quantified by the value of $\nu$, is large enough, then the instability could be overcome. To this extent viscosity might be seen to oppose instability in the super-critical region. However, this conclusion is derived solely within the linearised framework, and what is more, in the super-critical region the flow is largely laminar till the jump position is reached. Therefore, the true physical role of viscosity may not be of much significance in this region, which is dominated much more by nonlinearity. In that event, so far as the standing wave in the super-critical region is concerned, it would be reasonable to drop viscosity (by setting $\nu=0$ ) in Eq. (17), and retain only the time-dependent nonlinear effects on the background linearised stationary state. 
Having done so, employing the same mathematical methods and physical arguments that led to Eq. (12), and making use of the fact that $\tilde{f}^{01}=\tilde{f}^{10}$, a quadratic dispersion relation in $\omega$ can be derived from Eqs. (17) and (18), which will read as

$$
\omega^{2} \int p_{\omega}^{2}\left(v_{0}+\lambda \tilde{\mathrm{f}}^{00}\right) \mathrm{d} r+\mathrm{i} \lambda \omega \int p_{\omega}^{2} \frac{\partial \tilde{\mathrm{f}}^{00}}{\partial t} \mathrm{~d} r+\int v_{0}\left(v_{0}^{2}-c_{\mathrm{g}}^{2}\right)\left(\frac{\mathrm{d} p_{\omega}}{\mathrm{d} r}\right)^{2} \mathrm{~d} r-\lambda \int\left[p_{\omega} \frac{\mathrm{d} p_{\omega}}{\mathrm{d} r} \frac{\partial \tilde{\mathrm{f}}^{01}}{\partial t}-\tilde{\mathrm{f}}^{11}\left(\frac{\mathrm{d} p_{\omega}}{\mathrm{d} r}\right)^{2}\right] \mathrm{d} r=0 .
$$

It will be easy to conclude from this expression that the time-dependent part of the perturbation, given by $\exp (-\mathrm{i} \omega t)$, will have no complex part and, therefore, there will be a divergent mode of the perturbation arising solely due to nonlinearity.

While all this can be deduced about the amplitude-dependent phase (a regular feature where nonlinearity is concerned), it does not indicate anything precise about the behaviour of the amplitude of the perturbation itself. To do so it shall be necessary to adopt an approach that is quite common in studying amplitude equations [27, 28]. First the expression for $f^{\prime}(r, t)$ is to be modified slightly and written as $f^{\prime}(r, t)=\xi(t) p_{\omega}(r) \exp (-\mathrm{i} \omega t)$, with $\xi(t)$ being a slowly-varying function of time, containing information on the nonlinear time-dependent growth of the amplitude. The behaviour of $\xi(t)$ is to be studied at a fixed value of $r$ very close to the jump itself, where, as experimental evidence shows, the amplitude is saturated by nonlinear effects [17]. The justification for imposing the requirement of $\xi(t)$ being a slowly-varying function of time lies in the fact that the time scale for the amplitude amplification of the perturbation in the vicinity of the jump is much greater than the dynamic time scale for the formation of the jump, $r_{\mathrm{j}} / c_{\mathrm{g}}$.

Now Eqs. (15) and (16) connect $h^{\prime}$ and $v^{\prime}$, respectively, to $f^{\prime}$. Considering the modified expression of $f^{\prime}$, it should therefore be easy to appreciate that both $h^{\prime} \sim \xi(t)$ and $v^{\prime} \sim \xi(t)$. By this argument, since $\omega$ also contains $h^{\prime}$ and $v^{\prime}$, it should be noted that $\omega \sim \xi(t)$. Following this understanding, using the modified form of $f^{\prime}(r, t)$ in Eq. (17), and neglecting all second derivatives of $\xi(t)$, it can now be argued that the general form of the variation of $\xi(t)$ with respect to $t$ will be given by $\dot{\xi}=a \xi+b \xi^{3}$, with any even power of $\xi(t)$ being left out to preserve time-reversal symmetry, when $\xi \longrightarrow-\xi$. The coefficients $a$ and $b$ will determine the growth of $\xi$, and with $a>0$ there will be an early growth mode, but this growth will be saturated if $b<0$. That this is precisely what happens inside the super-critical region close to the jump, is very much in evidence through experimental studies [17]. With regard to this it would also be quite worthwhile to draw attention to the fact that for a shallow-layer flow of liquid helium, below its superfluid transition temperature, a very striking feature is the occurrence of a standing capillary wave (stationary ripples) between the impact point of the jet and the jump radius [15].

\section{TRAVELLING WAVES}

In treating the perturbation as radially propagating waves of very high frequency, a condition that is needed to be satisfied is that the frequency, $\omega$, of the travelling waves should be much greater than any characteristic frequency in the system. Since $t_{\mathrm{visc}}$ appears as a natural time scale in the system, it would be logical to use this time scale and impose the condition that $\omega \gg t_{\text {visc }}^{-1}$. The spatial part of the perturbation is prescribed as $p_{\omega}(r)=e^{s}$, with $s$ itself being given by the power series,

$$
s(r)=\sum_{n=-1}^{\infty} \omega^{-n} k_{n}(r) .
$$

The complete perturbation will be given as $f^{\prime}(r, t)=\exp (s-\mathrm{i} \omega t)$. Following some algebra, Eq. (11) can be written in a slightly altered form as

$$
\left(v_{0}^{2}-c_{\mathrm{g}}^{2}\right) \frac{\mathrm{d}^{2} p_{\omega}}{\mathrm{d} r^{2}}+\left[3 v_{0} \frac{\mathrm{d} v_{0}}{\mathrm{~d} r}-\frac{1}{v_{0}} \frac{\mathrm{d}}{\mathrm{d} r}\left(v_{0} c_{\mathrm{g}}^{2}\right)-2 \mathrm{i} v_{0} \omega+3 \nu \frac{v_{0}}{h_{0}^{2}}\right] \frac{\mathrm{d} p_{\omega}}{\mathrm{d} r}-\omega\left(\omega+2 \mathrm{i} \frac{\mathrm{d} v_{0}}{\mathrm{~d} r}+\mathrm{i} \nu \frac{1}{h_{0}^{2}}\right) p_{\omega}=0,
$$

in which $p_{\omega}(r)$ is substituted by making use of the power series given by Eq. (21). After this the three successive highestorder terms in $\omega$ will be obtained as $\omega^{2}, \omega$ and $\omega^{0}$, all of whose coefficients are collected and summed up separately, and each individual sum of coefficients is then set to zero. Solutions resulting from expressions involving $\omega^{2}$ and $\omega$, are given as

$$
k_{-1}=\int \frac{\mathrm{i}}{v_{0} \pm c_{\mathrm{g}}} \mathrm{d} r
$$

and

$$
k_{0}=-\frac{1}{2} \ln \left(v_{0} c_{\mathrm{g}}\right) \pm \nu \int \frac{1}{c_{\mathrm{g}} h_{0}^{2}}\left[1 \mp \frac{3}{2(\mathcal{F} \pm 1)}\right] \mathrm{d} r,
$$

respectively, with $\mathcal{F}$ being the Froude number, $\mathcal{F}=v_{0} / c_{\mathrm{g}}$.

It will be necessary to show that all successive terms of $s(r)$ will self-consistently follow the condition $\omega^{-n}\left|k_{n}(r)\right| \gg$ $\omega^{-(n+1)}\left|k_{n+1}(r)\right|$, i.e. the power series given by $p_{\omega}(r)$ will converge very rapidly with increasing $n$, so that the series can be 
truncated after the first few terms. This whole approach of studying high-frequency travelling waves, has also been successfully applied in astrophysical flows [22, 25]. The convergence criterion can be shown to be very much true for the radial shallowwater flow here, considering the behaviour of the flow in both the super-critical (where $v_{0} \sim$ constant and $h_{0} \sim r^{-1}$ ) and the sub-critical (where $v_{0} \sim r^{-1}$ and $h_{0} \sim$ constant) regions. In the inviscid limit the first three terms in $k_{n}(r)$ will behave as $k_{-1} \sim r, k_{0} \sim \ln r$ and $k_{1} \sim r^{-1}$. With the inclusion of viscosity as a physical effect, it can be seen from Eqs. (23) and (24), respectively, that while $k_{-1}$ remains unaffected, $k_{0}$ acquires a $\nu$-dependent term that goes asymptotically as $r$. Similarly, two such viscosity-dependent terms, going as $r$ and $\ln r$, respectively, are added to $k_{1}$. However, since $\nu$ has a relatively small value for water, and since the frequency of the travelling waves is at the same time very great (implied by $\omega \gg t_{\text {visc }}^{-1}$ ), the self-consistency requirement still holds. Therefore, it should be quite sufficient to truncate the power series expansion of $s(r)$ after considering the two leading terms only, and leave out $k_{1}$, which will anyway contibute only to the phase of the perturbation and not its amplitude. An expression for the perturbation will, therefore, be obtained as

$$
f^{\prime}(r, t) \simeq \frac{A_{ \pm}}{\sqrt{v_{0} c_{\mathrm{g}}}} \exp \left[\int\left(\frac{\mathrm{i} \omega}{v_{0} \pm c_{\mathrm{g}}} \pm \frac{\nu}{c_{\mathrm{g}} h_{0}^{2}}\left[1 \mp \frac{3}{2(\mathcal{F} \pm 1)}\right]\right) \mathrm{d} r\right] e^{-\mathrm{i} \omega t},
$$

which should be seen as a linear superposition of two waves with arbitrary constants $A_{+}$and $A_{-}$. Both these two waves move with a velocity, $c_{\mathrm{g}}$, relative to the fluid, one against the bulk flow and the other along with it, while the bulk flow itself has a velocity, $v_{0}$. All questions pertaining to the growth or decay in the amplitude of the perturbation will be crucially decided by the real terms derived from $k_{0}$. For the choice of the upper sign, i.e. for outgoing waves, the amplitude will be amplified as the wave propagates for a certain distance outwards, and then it will start decaying. A quantitative estimate can be made of the asymptotic behaviour of the perturbation. For the highly super-critical region of the flow, much before the jump, $v_{0}$ is approximately constant, while $h_{0} \sim r^{-1}$. The inviscid term in Eq. (24) will thus contribute a growth behaviour going as $r^{1 / 4}$. The growth of the viscous term on the other hand will vary as exp $\left[\left(r / r_{\mathrm{s} 1}\right)^{3.5}\right]$, with $r_{\mathrm{s} 1}$ being a suitable scale factor. At the opposite end, when the flow is in the highly sub-critical region, $h_{0}$ will be nearly constant, while $v_{0} \sim r^{-1}$. In this region the contribution of the inviscid term to the perturbation will be in the form of a growth going as $r^{1 / 2}$, which, however, will be suppressed by an exponential decay contributed by the viscous term, going approximately as $\exp \left(-r / r_{\mathrm{s} 2}\right)$, with $r_{\mathrm{s} 2}$ being another scale factor. Interestingly enough, in the vicinity of the jump, the scale factor, $r_{\mathrm{s} 2}$, reproduces the exact scaling form of the jump radius derived by Bohr et al. [9]. Quite evidently, the perturbation starts decaying after crossing the region where $\mathcal{F}=0.5$. However, even when the outgoing wave grows in amplitude, the growth is not unrestrained anywhere in the region of physical interest, and although it might disturb the jump itself somewhat while passing through it, the stability of the flow as well as the jump will not be too much adversely affected overall. So all that happens in the case of the outgoing wave is that starting from the super-critical region, it is completely transmitted through the jump discontinuity, continuing to grow in amplitude for some distance even beyond the jump, but decaying out eventually at very large distances.

This relatively placid state of affairs receives a severe jolt with the choice of the lower sign in Eq. (24), i.e. for incoming waves. It is evident from Eq. (24) that for an incoming wave progressing upstream from the highly sub-critical flow region to the vicinity of the jump (where the Froude number, $\mathcal{F}$, will be close to unity), the viscous term will cause a large exponential divergence in the amplitude of the perturbation, and the flow will therefore suffer noticeably from large fluctuations. On the other hand, in the super-critical region, it is because of the viscous term once again that the flow will be pronouncedly stable under any perturbation propagating upstream. Physically speaking this is what it should be anyway, because in the super-critical region the bulk flow proceeds much faster against the propagation of the perturbation, and since the super-critical region itself has only a finite spatial extent, the perturbation is not allowed to remain here for a span of time long enough to destabilise the flow in any way. Indeed, in the super-critical region just before the jump, the amplitude of the perturbation decays to vanishingly small values. This can only imply that any incoming perturbation propagating upstream from the sub-critical region is not allowed to be transmitted through the jump discontinuity into the super-critical region, and this is entirely in agreement with the white hole analogy presented at the end of Section $\amalg$.

This theoretical contention has received much support from an experimental work carried out by Kate et al. [18] to study hydraulic jumps formed due to adjacent normal impinging liquid jets. In a series of clearly illustrative photographs, these authors have recorded that when one of the two adjacent liquid jets is gradually moved along a straight line towards the other one, there will come a stage when, with the circular jumps formed by the two vertical jets being close enough to each other, the liquid trapped between the two jumps will be noticeably pushed up to stand at a much greater height than the rest of the flow. In a manner of speaking, a small wall of water — the liquid used in this experimental study — will be formed. It is easy to see that the jump formed by the moving jet behaves as a sub-critical disturbance propagating upstream towards the jump formed due to the static jet. This disturbance cannot penetrate through the static jump, and so as it approaches the jump region, its vertical height increases dramatically, due to accumulation of the liquid. This point has been amply demonstrated by the photograph (taken by Kate et al. [18]) in Figure 1. However, the disturbance propagating upstream in this experiment, is not a purely axisymmetric disturbance, and hence, there is a lack of symmetry in the formation of the wall of water between the two jumps. It is conceivable that if the perturbation propagating upstream were constrained to have been perfectly axisymmetric in nature, then one would have obtained a manifestly circular wall of water to be caught between the incoming waves and the periphery of the circular hydraulic jump. Relaxation for this situation can be achieved through the perturbation expending 


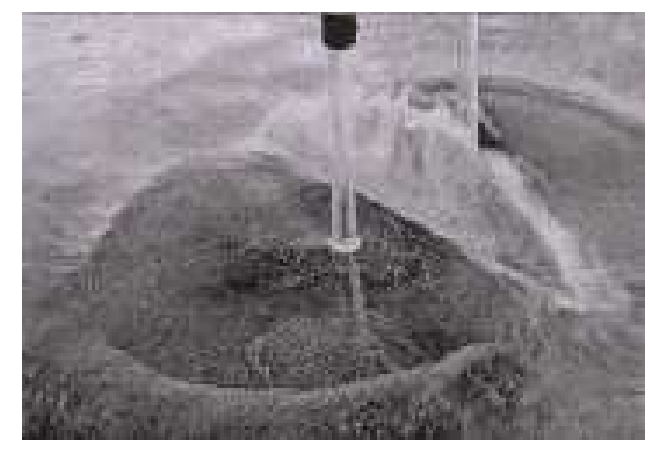

FIG. 1: An oblique view of the wall of water raised between two adjacent hydraulic jumps (Photo obtained by the courtesy of R. P. Kate, P. K. Das and S. Chakrabarti).

itself in destabilising the jump. That the flow rate (and connected to it by a scaling relation, the jump position itself) is highly destabilised by a travelling perturbation incident from the sub-critical side, is a feature that is in quite close correspondence with the observation of Hansen et al. [12] that the jump position starts fluctuating beyond a certain critical value of the flow rate.

The time-averaged energy flux associated with the travelling perturbation can be estimated by first noting that in an annular element of fluid, the energy arising due to the perturbation is given by the sum of its kinetic energy,

$$
\mathcal{E}_{\text {kin }}=2 \pi r \frac{\rho}{2}\left(h_{0}+h^{\prime}\right)\left(v_{0}+v^{\prime}\right)^{2} \mathrm{~d} r
$$

and its internal energy,

$$
\mathcal{E}_{\text {int }}=2 \pi r \rho\left[\epsilon h_{0}+h^{\prime} \frac{\partial}{\partial h_{0}}\left(h_{0} \epsilon\right)+\frac{1}{2} h^{\prime 2} \frac{\partial^{2}}{\partial h_{0}^{2}}\left(h_{0} \epsilon\right)\right] \mathrm{d} r
$$

in which $\rho$ is the constant density of the fluid, and $\epsilon$ is the internal energy per unit mass [2]. The first-order terms all vanish upon time-averaging, leaving an expression for the energy of the perturbation that involves only second-order terms. This reads as

$$
\mathcal{E}_{\text {pert }}=2 \pi r \frac{\rho}{2}\left[h_{0} v^{\prime 2}+2 v_{0} h^{\prime} v^{\prime}+{h^{\prime}}^{2} \frac{\partial^{2}}{\partial h_{0}^{2}}\left(h_{0} \epsilon\right)\right] \mathrm{d} r
$$

The height fluctuation, $h^{\prime}$, and the velocity fluctuation, $v^{\prime}$, could both be expressed in terms of $f^{\prime}$, by making use of Eqs. (4) and (3), respectively. Carrying out a time-averaging over the periodic part of the square of $f^{\prime}$, under the approximation that to a leading order $s \simeq \omega k_{-1}$, will lead to an expression for the energy flux of the propagating perturbation as

$$
\mathrm{F}_{\text {pert }} \simeq \frac{\pi^{2} \rho}{Q} \frac{A_{ \pm}^{2}}{\mathcal{F} \pm 1}\left[1 \pm 2 \mathcal{F}+\frac{1}{g} \frac{\partial^{2}}{\partial h_{0}^{2}}\left(h_{0} \epsilon\right)\right] \exp \left( \pm 2 \nu \int \frac{1}{c_{\mathrm{g}} h_{0}^{2}}\left[1 \mp \frac{3}{2(\mathcal{F} \pm 1)}\right] \mathrm{d} r\right),
$$

with a factor of $1 / 2$ deriving from the time-averaging. Once again it can be seen that the incoming wave will cause much destabilisation for the energy flux in the region where the value of the Froude number, $\mathcal{F}$, approaches unity.

\section{CONCLUDING REMARKS}

Some general comments would well be in order here, considering the broad appeal that the physical problem of the hydraulic jump holds across various apparently unrelated disciplines. In this paper a physical explanation for the hydraulic jump has been furnished with the help of the analogy of an acoustic white hole, and by applying standard mathematical tools from stability studies in astrophysical accretion. In doing so the most salient fact that has been unearthed is that the invariance required to invoke the analogy of acoustic geometry holds good even when nonlinearity is taken into account. So this whole study has amounted to a very striking convergence of fluid dynamics (both laboratory and astrophysical), analogue gravity and the mathematics of nonlinear phenomena.

The reverse aspect of this condition is also true. Recent literature has found mention of astrophysical problems being addressed in terms of the analogy of a hydraulic jump. The radial profiles of large gaseous baryonic structures, such as galaxies and galaxy clusters, follow one power law in the inner part, and a different one in the outer part, beyond a certain characteristic radius. 
This transition has been seen to be sharp. It does not necessarily imply that the two distinctly different profiles are premised on different kinds of physical properties. Rather, very much like the hydraulic jump, the governing equations will have two solutions, and both will be availed of simultaneously [29]. Another related astrophysical connection pertains to spiral shocks in protoplanetary discs. Spiral shocks, for most protoplanetary discs, create a loss of vertical equilibrium in the post-shock region, and this results in a rapid expansion of the gas in a direction that is perpendicular to the plane of the disc. This expansion has characteristics that are similar to hydraulic jumps [30].

While these facts are by now well established, the present work may also broach a related issue. In astrophysics, the question of merging black holes is presently being much pondered over. Some light could be cast on this question by the kind of experiment conducted by Kate et al. [18], which has provided support for some of the theoretical arguments in this paper. The collision of two adjacent circular hydraulic jumps, both modelled as acoustic white holes, could provide a laboratory test case for the cognate situation of merging astrophysical black holes.

\section{Acknowledgments}

The authors are grateful to Prasanta K. Das, Rama Govindarajan and Steen H. Hansen for many helpful comments. The authors are also indebted to Tapas K. Das for drawing attention to some recent works in the subject of analogue gravity, and to Ajit K. Kembhavi for extending unstinted support in various respects.

[1] C. Walshaw and D. A. Jobson, Mechanics of Fluids (Longman, London, 1979).

[2] L. D. Landau and E. M. Lifshitz, Fluid Dynamics (Butterworth-Heinemann, Oxford, 1987).

[3] S. Granger, Engineering Fluid Mechanics (Dover, New York, 1987).

[4] T. E. Faber, Fluid Dynamics for Physicists (Cambridge University Press, Cambridge, 1995).

[5] E. Guyon, J.-P. Hulin, L. Petit, and C. D. Mitescu, Physical Hydrodynamics (Oxford University Press, Oxford, 2001).

[6] I. Tani, J. Phys. Soc. Japan 4, 212 (1949).

[7] E. J. Watson, J. Fluid Mech. 20, 481 (1964).

[8] R. G. Olsson and E. T. Turkdogan, Nature 211, 813 (1966).

[9] T. Bohr, P. Dimon, and V. Putkaradze, J. Fluid Mech. 254, 635 (1993).

[10] T. Bohr, C. Ellegaard, A. E. Hansen, and A. Hanning, Physica B 228, 1 (1996).

[11] T. Bohr, V. Putkaradze, and S. Watanabe, Phys. Rev. Lett. 79, 1038 (1997).

[12] S. H. Hansen, S. Hørlück, D. Zauner, P. Dimon, C. Ellegaard, and S. C. Creagh, Phys. Rev. E 55, 7048 (1997).

[13] J. W. M. Bush and J. M. Aristoff, J. Fluid Mech. 489, 229 (2003).

[14] M. Mathur, R. DasGupta, N. R. Selvi, N. S. John, G. U. Kulkarni, and R. Govindarajan, Phys. Rev. Lett. 98, 164502 (2007).

[15] E. Rolley, C. Guthmann, and M. S. Pettersen, Physica B 394, 46 (2007).

[16] S. B. Singha, J. K. Bhattacharjee, and A. K. Ray, Eur. Phys. J. B 48, 417 (2005).

[17] G. E. Volovik, J. Low Temp. Phys. 145, 337 (2006).

[18] R. P. Kate, P. K. Das, and S. Chakrabarti, J. Fluid Mech. (To appear) (2007).

[19] R. Schützhold and W. Unruh, Phys. Rev. D 66, 044019 (2002).

[20] C. Barceló, S. Liberati, and M. Visser, gr-qc/0505065.

[21] G. E. Volovik, JETP Letters 82, 624 (2005).

[22] J. A. Petterson, J. Silk, and J. P. Ostriker, MNRAS 191, 571 (1980).

[23] T. Theuns and M. David, ApJ 384, 587 (1992).

[24] A. K. Ray, MNRAS 344, 1085 (2003).

[25] S. Chaudhury, A. K. Ray, and T. K. Das, MNRAS 373, 146 (2006).

[26] W. Unruh and R. Schützhold, Phys. Rev. D 71, 024028 (2005).

[27] S. H. Strogatz, Nonlinear Dynamics and Chaos (Addison-Wesley Publishing Company, Reading, MA, 1994).

[28] D. W. Jordan and P. Smith, Nonlinear Ordinary Differential Equations (Oxford University Press, Oxford, 1999).

[29] S. H. Hansen and J. Stadel, ApJ 595, L37 (2003).

[30] A. C. Boley and R. H. Durisen, ApJ 641, 534 (2006). 